\title{
Deterministic System Identification Using RBF Networks
}

\author{
Joilson Batista de Almeida Rego, ${ }^{1,2}$ Allan de Medeiros Martins, ${ }^{3}$ and Evandro de B. Costa ${ }^{2}$ \\ ${ }^{1}$ Postgraduate Program in Electrical and Computer Engineering (PPgEEC), LACI/DEE, Federal University of Rio Grande do Norte, \\ 59078-970 Natal, RN, Brazil \\ ${ }^{2}$ The Computer Science Institute at the Federal University of Alagoas (UFAL), 57072-900 Maceió, AL, Brazil \\ ${ }^{3}$ Department of Electrical Engineering (DEE), Federal University of Rio Grande do Norte, 59078-970 Natal, RN, Brazil
}

Correspondence should be addressed to Joilson Batista de Almeida Rego; jotarego@gmail.com

Received 5 December 2013; Revised 19 February 2014; Accepted 12 March 2014; Published 30 April 2014

Academic Editor: Chien-Yu Lu

Copyright (c) 2014 Joilson Batista de Almeida Rego et al. This is an open access article distributed under the Creative Commons Attribution License, which permits unrestricted use, distribution, and reproduction in any medium, provided the original work is properly cited.

\begin{abstract}
This paper presents an artificial intelligence application using a nonconventional mathematical tool: the radial basis function (RBF) networks, aiming to identify the current plant of an induction motor or other nonlinear systems. Here, the objective is to present the RBF response to different nonlinear systems and analyze the obtained results. A RBF network is trained and simulated in order to obtain the dynamical solution with basin of attraction and equilibrium point for known and unknown system and establish a relationship between these dynamical systems and the RBF response. On the basis of several examples, the results indicating the effectiveness of this approach are demonstrated.
\end{abstract}

\section{Introduction}

System identification is the process of deriving a mathematical model of a system using observed data [1]. Once we have the mathematical model of a system we can analyze it (plant or process); however the representation of a physical system is a difficult task. To mitigate this difficulty, an alternative approach is to build models from measured or observed data.

It is important to understand that the model developed on the identification of a certain system represents its approximation; in this paper a mathematical model that represents the real physical system as close as possible is presented. The achievement of such a model is needed for the majority of industrial processes, since knowing the system allows us to control it, therefore the necessity of the identification process.

If the identification is based exclusively on the group of data obtained through measurements and assuming that there is no knowledge of the system dynamics, the identification process is known as the black-box modeling; however, if the laws of physics that conduct the process are used to build a phenomenological model of the system, we have the white-box modeling. Thus certain knowledge about the system is used to improve the empirical modeling; we have a gray-box modeling. Thereby some understanding of the system can facilitate the identification, such as the order of the system, response, sampling time, stability, range, delay time, freedom degree, and basic characteristics of nonlinearity [1]. In many cases we use identification techniques to obtain models that describe the behavior of a system. With such models we intend to describe the relation cause/effect between the input/output variables.

The motivation for the study of system identification techniques comes with the fact that usually we do not know the equations that describe the dynamics of such system and to obtain these equations, depending on the complexity of the system, would be impractical due to time and resources limitations. System identification consists in choosing an appropriate model and adjusting the parameters of the model according to some adaptive law such that the model's response to an input signal approximates the response of the real system to the same input. System identification is important not only for understanding and predicting the behavior of the process, but also for designing effective control laws [2]. 
In this study we intend to investigate and analyze the identification of a nonlinear system using a RBF network. The RBF networks are a particular class of function approximators which can be trained, using a set of samples. RBFs have been receiving a growing amount of attention since their initial proposal by Broomhead and Lowe [3].

Other major contributions to the theory, design, and applications of RBF networks can be found, for instance, in papers by Moody and Darken and Poggio and Girosi [4-6]. Poggio and Girosi explain the use of regularization theory applied to this class of neural networks as a method for improved generalization to new data. The applications of RBF networks in identification and control have been demonstrated, through simulation, by several studies, including [79].

We present a design procedure, for identification of a nonlinear dynamical system using RBF networks. This study also serves to unify kernel methods and system identification and control theory. In Section 2 the motivation for use the Radial Basis Function networks in approximation theory and data modeling is presented. In Section 3 our problem formulation leads to a system identification based on the use of Radial Basis Function networks and the results are used to show that a proposed combination of static RBF networks with dynamical components forms a recurrent network capable of approximating a large class of dynamical systems. The recurrent network is used for describing the dynamics of a nonlinear system (induction machine) or an autonomous dynamic system. This model has sufficient information to predict the system evolution for many inputs. Finally, in Section 4 we discuss the contribution of the paper and draw some final conclusions.

\section{Radial Basis Function Neural Networks}

Methods of regression and function approximation have gained more attention recently. Not only in math, but also in different areas of knowledge such as engineering, geophysics, signals and images processing, graphic computing, theory of machine learning, neural networks, and data mining, among others.

The function approximation theory consists in approximating a multivariate function $y(\cdot)$ by an approximation function $\widehat{f}(\mathbf{w}, \mathbf{x})$, given a fixed number of parameters $\mathbf{w}$, where $\mathbf{x}$ and $\mathbf{w}$ are vectors. Thus, there are two important aspects of the model to be defined: the function $\widehat{f}(\cdot)$ and the parameters of the vector $\mathbf{w}$. By choosing the specific $\hat{f}$, the problem is reduced to determiningthe vector $\mathbf{w}$, which offers a better function approximation $y(\cdot)$ for a group of data $\mathbf{x}$.

The identification strategy used in RBF networks consists in approximating an unknown function with a linear combination of a group of nonlinear functions, known as base functions, in order to predict the value of one or more objective variables, given a set of input variables [10].

The radial basis functions can be seen as a mathematical structure whose objective is to approximate disperse data in multiple dimensions with precision; this approach is frequently applied in the theory of modern approximation

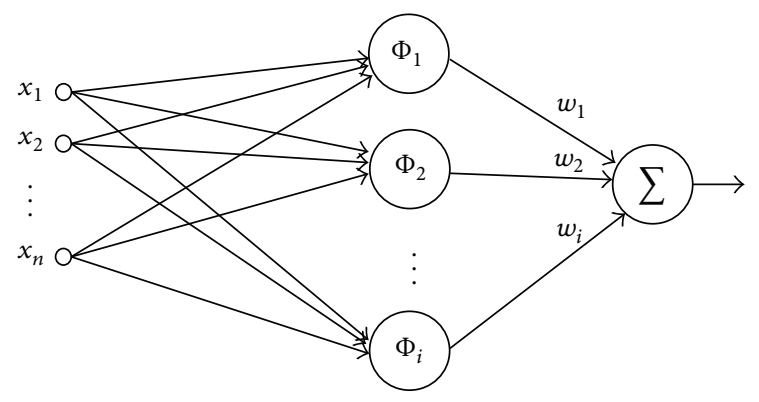

FIgURE 1: Example of the RBF topology in which $x_{1}, x_{2}, \ldots, x_{n}$ represent the input data, $\Phi_{1}, \Phi_{2}, \ldots, \Phi_{i}$ the basis functions, and $w_{1}, w_{2}, \ldots, w_{i}$ the parameters of the model.

[11]. This approach is very useful in the modeling and identification of nonlinear systems. There are many types of basis functions, such as multiquadrics, Cauchy, and Gaussians, among others [10].

The basis functions used in this work are radials; then we can define a function $\Phi: \mathbb{R}^{n} \rightarrow \mathbb{R}$ if a univariate function $\Phi:[0, \infty) \rightarrow \mathbb{R}$ exists, such that

$$
\Phi(\mathbf{x})=\varphi(r), \quad \text { with } \quad r=\|\mathbf{x}-\mathbf{c}\|,
$$

where $\|\cdot\|$ is the Euclidean norm. In other words, we can say that the value of $\Phi$ at any point has a fixed distance to the constant origin (or another fixed point named center c). Therefore, $\Phi$ is radially (or spherically) symmetric around the center. In definition (1) we used a function based on the Euclidean distance to approach another case of radial basis function, which will be shown next. The radial basis functions networks can be represented in Figure 1.

In our analysis we consider RBF networks with Gaussian activation functions.

2.1. Gaussian Radial Basis Functions. The Gaussian functions are local functions and are widely used in the theory of multivariate functions approximation, usually expressed as

$$
\Phi(r)=g(r)=\exp \left(-\frac{r^{2}}{2 \sigma^{2}}\right) .
$$

In this type of function the $\sigma$ parameter corresponds to the width of the Gaussian function; in other words, it defines the average Euclidean distance (average radius) which measures the dispersion of the data represented by the function of radial basis around its center [12]. The $\sigma$ parameter represents how quickly the value of the radial basis function falls to zero, as the input data departs from the center.

The networks using Gaussian radial basis functions produce a nonlinear mapping of the input space into a space of characteristics, usually of high dimensionality, followed by a linear mapping of the hidden space for the output space. This nonlinear transformation is defined by the radial basis functions and the group of weights defines the linear transformation. In Figure 2 we can observe that the Gaussian basis functions are crescent localized functions at $x=-\infty \rightarrow$ $x=0$ and decrescent at $x=0 \rightarrow x=\infty$. 


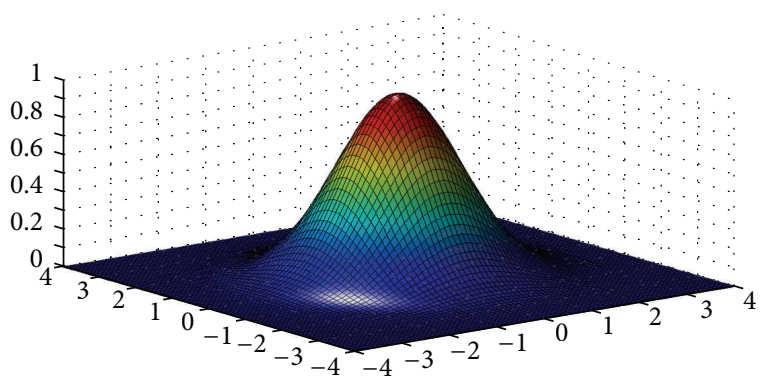

FIGURE 2: Example of a bidimensional radial basis function with $\sigma=$ 1 centered on the origin.

Another important concept related to the Gaussian radial basis functions is that they are defined positive for each parameter $\sigma>0$. A function is defined positive if the quadratic form

$$
\sum_{i=1}^{n} \sum_{j=1}^{n} G\left(x_{i}-c_{j}\right)>0
$$

for $n$ distinct pairs of different points $x_{1}, x_{2}, \ldots, x_{n}$ and $c_{1}, c_{2}, \ldots, c_{n}[12]$.

We can observe that the design of a network using radial basis functions can be seen as a problem of curve adjustment, where the learning is equivalent to find a surface in a multidimensional space, mapping the results in the better adjustment to a group of training data.

2.2. Learning Algorithms. Assuming a group of training data, we need to estimate a function $f$ as close as possible to the objective function $y$ using a RBF network. The universal approximation property states that an optimal solution to the approximation problem exists: finding the corresponding cost function minimum is the goal of the learning algorithms. There are different learning strategies that we can follow in the design of a RBF network, depending on how the centers of the $\mathrm{RBF}$ network are specified.

The simplest approach is to assume fixed Gaussian functions defining the activation functions of the hidden units. In particular, the centers locations may be chosen randomly from the training data set and kernel size is fixed according to the spread of the centers. The kernel size of all the Gaussian radial basis functions is fixed at

$$
\sigma=\frac{d}{\sqrt{2 n_{c}}},
$$

where $d$ is the maximum distance between the chosen centers and $n_{c}$ the number of centers [13].

Another method is the Gradient Descent Optimization used in $[14,15]$. For the supervising learning strategies, the RBF network optimization is usually based on a gradient descent learning process that is generally suffered from the local minima problem and slow convergence speed. In this paper, a modified learning scheme is used for computing the adjustable (weight) parameters [13]; the cost functional of this problem is convex with respect to the linear parameters $w_{i}$.
The optimum values of these parameters are determined by the linear least squares method directly with regularization parameter so that the algorithm convergence is speeded up. The training structure is not initially given, so in the regularization theory the function approximation problem is to find the function $\widehat{f}(x)$ that minimizes

$$
J=\frac{1}{2} \sum_{i=1}^{n}\left[y_{i}-\widehat{f}\left(x_{i}\right)\right]^{2}+\frac{1}{2} \lambda\|D \widehat{f}\|^{2},
$$

where $\lambda>0$ and $\lambda \in \mathbb{R}$ are called the regularization parameter, $J$ is the Tikhonov functional, and $D$ is differential operator. Differentiating (5) we obtain the Euler-Lagrange equation for $J$ :

$$
D^{*} D \widehat{f}(x)=\frac{1}{\lambda} \sum_{i=1}^{n}\left[y_{i}-\widehat{f}\left(x_{i}\right)\right] \delta\left(x-c_{i}\right)
$$

Since $\delta(\cdot)$ is the Dirac delta function using its shifting property, the solution $f$ in $(6)$ is

$$
\widehat{f}(x)=\frac{1}{\lambda} \sum_{i=1}^{n}\left[y_{i}-\widehat{f}\left(x_{i}\right)\right] G\left(x, c_{i}\right) .
$$

Equation (7) states that minimizing the solution $\widehat{f}(x)$ to the regularization problem is a linear superposition of Green functions. The shape of these functions depends only on the differential operator $D$, that is, on the assumptions concerning the mapping characteristics between input and output space. Thus the choice of $D$ determines the approximator basis functions. In particular if $D$ is invariant for rotation and translation the Green function is [13]

$$
G\left(x, c_{i}\right)=G\left(x-c_{i}\right)
$$

$c_{i}$ represents the expansion center and the term $w_{i}=$ $(1 / \lambda) \sum_{i=1}^{n}\left[y_{i}-\widehat{f}\left(x_{i}\right)\right]$ represents the coefficient expansion. So the minimized solution of (7) evaluated in the point $x_{j}$ is

$$
\widehat{f}(x)=\sum_{i=1}^{n} w_{i} G\left(x_{j}, c_{i}\right), \quad j=1, \ldots, n .
$$

Evaluating (9), we get a system of $n$ simultaneous equations:

$$
\begin{gathered}
\widehat{f}_{1}=w_{1} \exp \left[-\frac{\mathbf{x}_{1}-\mathbf{c}_{1}}{2 \sigma^{2}}\right]+\cdots+w_{n} \exp \left[-\frac{\mathbf{x}_{1}-\mathbf{c}_{n}}{2 \sigma^{2}}\right] \\
\widehat{f}_{2}=w_{1} \exp \left[-\frac{\mathbf{x}_{2}-\mathbf{c}_{1}}{2 \sigma^{2}}\right]+\cdots+w_{n} \exp \left[-\frac{\mathbf{x}_{2}-\mathbf{c}_{n}}{2 \sigma^{2}}\right] \\
\vdots \\
\widehat{f}_{n}=w_{1} \exp \left[-\frac{\mathbf{x}_{n}-\mathbf{c}_{1}}{2 \sigma^{2}}\right]+\cdots+w_{n} \exp \left[-\frac{\mathbf{x}_{n}-\mathbf{c}_{n}}{2 \sigma^{2}}\right],
\end{gathered}
$$


where $\mathbf{c}=\left[c_{1}, c_{2}, \ldots, c_{n}\right]^{T}$ represents the vector of centers chosen from the group of input data; the equations above can be written in a shorter way as

$$
\begin{gathered}
g_{11} w_{1}+g_{12} w_{2}+\cdots+g_{1 n} w_{n}=\widehat{f}_{1} \\
g_{21} w_{1}+g_{22} w_{2}+\cdots+g_{2 n} w_{n}=\widehat{f}_{2} \\
\vdots \\
g_{n 1} w_{1}+g_{n 2} w_{2}+\cdots+g_{n n} w_{n}=\widehat{f}_{n} .
\end{gathered}
$$

Where $g_{i j} i, j=1, \ldots, n$ is the Gaussians radial basis functions, $w_{i}$ is the parameters of the model, the equations system above can be written in matrix form as

$$
\begin{aligned}
& {\left[\begin{array}{c}
\widehat{f}_{1} \\
\widehat{f}_{2} \\
\vdots \\
\widehat{f}_{n}
\end{array}\right]} \\
& =\left[\begin{array}{ccc}
g_{11}=\exp \left[-\frac{\mathbf{x}_{1}-\mathbf{c}_{1}}{2 \sigma^{2}}\right] & \cdots & g_{1 n}=\exp \left[-\frac{\mathbf{x}_{1}-\mathbf{c}_{n}}{2 \sigma^{2}}\right] \\
\vdots & \vdots & \vdots \\
g_{n 1}=\exp \left[-\frac{\mathbf{x}_{n}-\mathbf{c}_{1}}{2 \sigma^{2}}\right] & \cdots & g_{n n}=\exp \left[-\frac{\mathbf{x}_{n}-\mathbf{c}_{n}}{2 \sigma^{2}}\right]
\end{array}\right] \\
& \times\left[\begin{array}{c}
w_{1} \\
w_{2} \\
\vdots \\
w_{n}
\end{array}\right] .
\end{aligned}
$$

That is,

$$
\left[\begin{array}{c}
\widehat{f}_{1} \\
\widehat{f}_{2} \\
\vdots \\
\widehat{f}_{n}
\end{array}\right]=\left[\begin{array}{cccc}
g_{11} & g_{12} & \cdots & g_{1 n} \\
g_{21} & g_{22} & \cdots & g_{2 n} \\
\vdots & \vdots & \ddots & \vdots \\
g_{n 1} & g_{n 2} & \cdots & g_{n n}
\end{array}\right]\left[\begin{array}{c}
w_{1} \\
w_{2} \\
\vdots \\
w_{n}
\end{array}\right] .
$$

In the compact form, we have

$$
\mathbf{G} \cdot \mathbf{W}=\widehat{\mathbf{f}},
$$

where the system matrix $\mathbf{G}$ has inputs $g_{i j}$ and $\mathbf{W}$ represents the unknown coefficients vector (weights) defined by

$$
\mathbf{W}=\frac{1}{\lambda}(\mathbf{y}-\widehat{\mathbf{f}}) \text {. }
$$

Substituting (14) in (15), where $\mathbf{y}$ is the vector of desired values, we have

$$
\begin{gathered}
\mathbf{w}=\frac{1}{\lambda}(\mathbf{y}-\mathbf{G} \cdot \mathbf{W}) \\
\mathbf{w}+\frac{1}{\lambda} \mathbf{G} \cdot \mathbf{W}=\frac{1}{\lambda} \mathbf{y} \\
(\mathbf{G}+\lambda \mathbf{I}) \mathbf{W}=\mathbf{y} .
\end{gathered}
$$

In 1986, Micchelli established a relation between disperses data interpolation and positive defined functions. Micchelli demonstrated that, for a class of radial basis functions, the interpolation matrix $\mathbf{G}$ is nonsingular as long as the data are distinct. This is all that is demanded for the nonsingularity of the interpolation matrix [13].

There are many radial basis functions that are covered by the studies presented by Micchelli, the Gaussian among them, which lead us to conclude that, if we have Gaussian basis functionsgenerating an interpolation matrix $\mathbf{G}$, it will be defined as positive matrix, consequently having a nonsingular matrix. It may occur that the interpolation matrix $\mathbf{G}$ is not square; in this case, it is necessary to use the pseudoinverse matrix in the adjustment problem solution through the least squares. Then we have

$$
\mathbf{G}^{+} \mathbf{f}=\mathbf{w}
$$

where $\mathbf{G}^{+}=\left(\mathbf{G}^{T} \mathbf{G}\right)^{-1} \mathbf{G}^{T}$ represents the pseudoinverse of the matrix $\mathbf{G}$.

We can still add a parameter $\lambda$ to avoid conditioning problems in the interpolation matrix, in other words

$$
\mathbf{G}^{+}=\left(\mathbf{G}^{T} \mathbf{G}+\lambda \mathbf{I}\right)^{-1} \mathbf{G}^{T}
$$

so that

$$
\mathbf{w}=\mathbf{G}^{+} \mathbf{f} \text { for } \lambda=0 \text {. }
$$

When the parameter of regularization $\lambda$ is close to zero, the weights vector $\mathbf{w}$ converges to a solution of minimum norm for the vector error [13].

Using nonlinear basis functions, we allow the $\widehat{f}(\mathbf{w}, \mathbf{x})$ function to be a nonlinear function from the input data. The great advantage of functions (5) or (6), which are linear in $\mathbf{w}$, is the simplicity of the model, that is, the possibility of obtaining a linear combination in a closed form, through the application of solution techniques based on the least square method. The main problem in a RBF network is the choice of the centers and other parameters, inherent to the structure, for obtaining the best weights group. In the construction of a RBF network, we must take into account some parameters: the radius of the Gaussians $\sigma$, the number of centers, the input vector $\mathbf{x}$, and the value of the weights $\mathbf{w}$.

The three first requirements, the parameter $\sigma$ determination, the number of centers, and the determination of the inputs group, are based on heuristics, while the determination of $\mathbf{w}$ is done through the minimization of a cost functional. All the parameters must be chosen to minimize the training error through the adjustment of $\mathbf{w}[16]$.

In this study, the choice of the centers was made from a group of data extracted from the group of input using $k$ means algorithm for finding clusters and cluster centers in a set of unlabeled data and is computationally efficient [17] and the parameter $\sigma$ was adjusted using the Silverman rule [18]. The centers and the parameter $\sigma$ of the radial basis function are the parameters of the model, and the parameter $\sigma$ controls the smooth of the interpolation. All tests have been made without the use of the regularization parameter $\lambda$ defined in (5). The advances in RBF network training during the last couple decades can be seen in many studies [19-21]. 
2.3. The Induction Machine. The induction machine is the most common alternate current (AC) motor, mostly due to its simplicity, robust construction, and low cost. The name induction machine is derived from the fact that the alternating currents are induced in the rotor's circuit, by the stator magnetic field.

For the identification of an induction machine current plant, we will use a frequency inverter as an element for exciting the motor; in other words, the signal that feeds the machine is modulated in pulses width (PWM: Pulse Width Modulation) [22].

The drive module can be divided in two parts: induction machine and frequency converter. The platform induction machine has the following attributes:

(i) three-phase induction machine;

(ii) power: $1,5 \mathrm{CV}$;

(iii) number of poles: 4;

(iv) speed: $1720 \mathrm{rpm}$;

(v) voltage: $220 \mathrm{~V} / 380 \mathrm{~V}$;

(vi) current: 4,43 A/1,56 A.

The frequency converter is made of five parts: rectifier, capacitive filter, preload circuit, drivers, and inverter. The rectifier transforms the three-phased alternate voltage (which feeds the module) into a continuous voltage, which is filtered by the capacitors. The preload circuit allows the currents peak reduction in the initial load of the filter capacitors. The platform is configured to work at $220 \mathrm{~V}$, operating with the motor in triangle.

The PWM inverter is composed of a module containing a IGBTs (Insulated Gate Bipolar Transistor), which receive the actuation command through the PWM modulation generated by the interface. The measure acquisition system is composed of an analog-digital signal conversion board, which allows the acquisition of signals necessary to the proposed identification. The measured signals are currents and three-phase voltages.

\section{Results and Discussion}

In this section, we present the application of the proposed identification technique (RBF) in the dynamic system analysis (the current plant of the induction motor) based on observed data. Initially, the obtained data from the plant were used for validating and analyzing the model. The data used in the identification process were collected at LACI, Laboratory of Actuation Control and Instrumentation (UFRN), using current sensors.

The current plant of the induction machine is a nonlinear system of discrete time with discrete data; we assume that the nonlinearity of the system is unknown.

The tool used in the identification process is a neural network that uses a Gaussian radial basis function that gradually grows to encompass the adequate and sufficient space in a way that provides an approximation of the system. The $\mathrm{RBF}$ is an alternate tool for the solving problems in

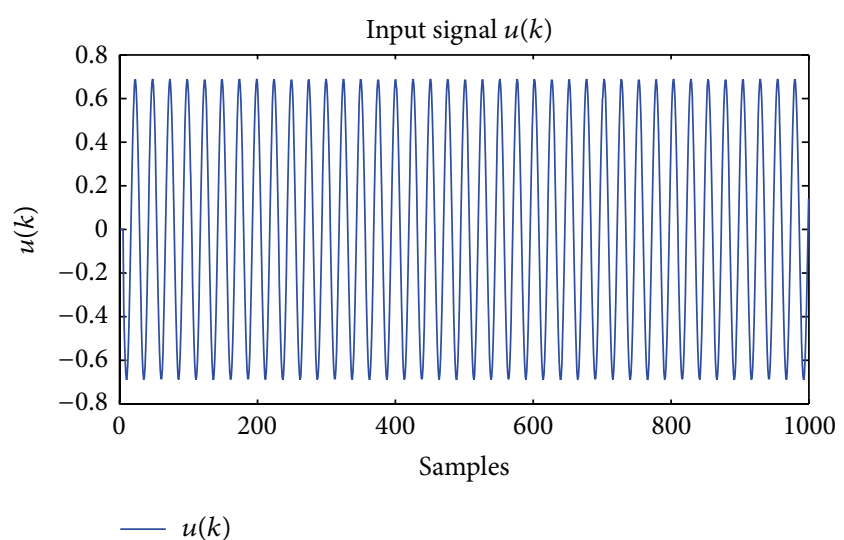

FIGURE 3: Sinusoidal signal applied in the plant due to the number of samples; in this case, for a better visualization, 1000 samples have been used in this graphic.

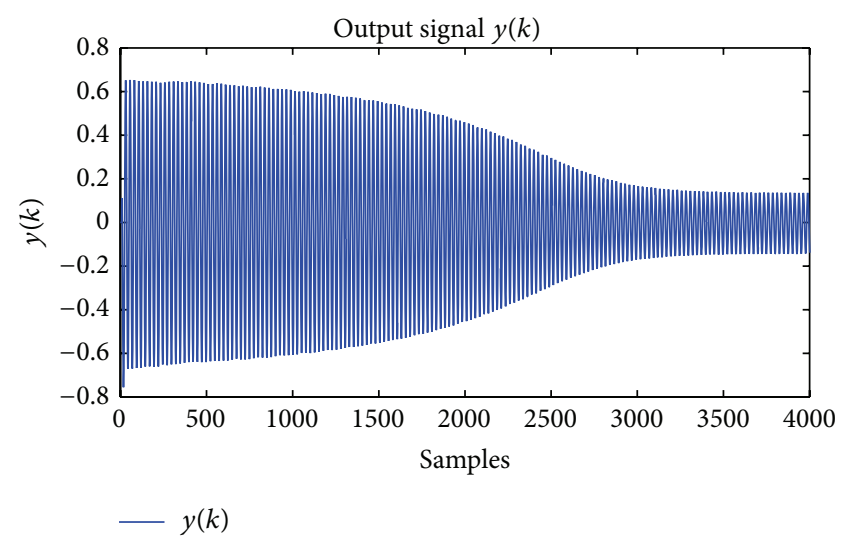

FIGURE 4: Response signal of the plant; in this case, for a better visualization 4000 samples have been used in this graphic.

identification and control, especially for those involving nonlinearities.

The system to be identified has the following attributes.

(i) It is a discrete time system.

(ii) It is primarily meant for the black box identification.

(iii) They are monovariables or SISO, Single Input Single Output, although all cases can be extended to more than one input and/or output.

The dynamic and static characteristics of a system will come out from its excitation signal; in this case the frequencies range of a signal must be carefully chosen when the objective is to excite the linear or nonlinear dynamics of a determined system. However, the amplitude or excursion of a signal is what takes a nonlinear system to achieve different points of operation revealing its nonlinear attributes. We have used as input a sinusoidal signal as shown in Figure 3.

And we have obtained the response signal shown in Figure 4.

In this work, the sampling period of $250 \mathrm{~ms}$ (milliseconds) was chosen to generate a good response. 
3.1. Determination of the Structure Models. If we consider the plant as a nonlinear function defined by

$$
\begin{gathered}
y(k+1)=f[y(k), y(k-1), y(k-2), u(k), \\
u(k-1), u(k-2)],
\end{gathered}
$$

where $[u(k), y(k)]$ represents the pair of inputs-output of the SISO plant in the moment $k$. This is a general model in which the output for any moment is the nonlinear function of the values givenby the inputs and outputs. The discrete representation of the system states can be defined as

$$
\mathbf{x}(k+1)=f(\mathbf{x}(k), \mathbf{u}(k)),
$$

where $f$ is an unknown function and is nonlinear in the parameters $x$ and $u$. If the function $f$ has a derivate around an arbitrary equilibrium point, then the nonlinear system defined in (20) can be approximated by a RBF network.

Results obtained by Powell [23] and Wendland [24] demonstrate that the RBF networks have the property to be a universal function approximators. More precisely, for a determined tolerance $\varepsilon$ admitted for an estimator and an arbitrary nonlinear function $f(\cdot)$, there will be a parameters vector $\mathbf{w}$ and a matrix $\mathbf{G}$, so that

$$
\max \left|f(x)-\mathbf{w}^{T} \mathbf{G}\right| \leq \varepsilon .
$$

This result is the base to justify the use of RBFs in nonlinear systems identification problems. The RBF universal approximation property guarantees the existence of these weights vector (parameters) $\mathbf{w}$ which minimize the average error between the system response and the RBF response, so the system can be represented by

$$
y(k+1) \cong f_{\mathrm{RBF}}\left(x_{i}, c_{j}, \mathbf{w}, \sigma\right),
$$

where $\mathbf{x}=[u(k), u(k-1), y(k-1), y(k-2))]$ is the RBF input vector, $\mathbf{c}$ the centers vector chosen from the input group using $k$ means algorithm [17], and $\sigma$ the width of the basis functions that is adjusted using the Silverman rule [18].

The main problem of the identification algorithm is to find the parameters vector $\mathbf{w}$ and the function that will build the approximate model for (20). In this paper we will work the identification problem in two different tasks.

First we will find the vector $\mathbf{w}$ that promotes the best approximation of the desired function through the RBF training using least squares and then we will analyze its dynamic behavior.

Based on some previous knowledge about the plant a model is chosen to identify it. In this study we will be considering this as being a second-order system. The goal is to determine the RBF weights using the least squares method. Figure 5 was implemented.

Analyzing the structure above it can be seen that the data presented to the RBF network is the data from the plant, and the estimated outputs of the RBF are compared with the plant outputs for calculating the average error used in the RBF network free parameters adjustment. In the training $40 \%$ of samples were used. This training process is offline and is based on the least squares method presented in the regularization theory.

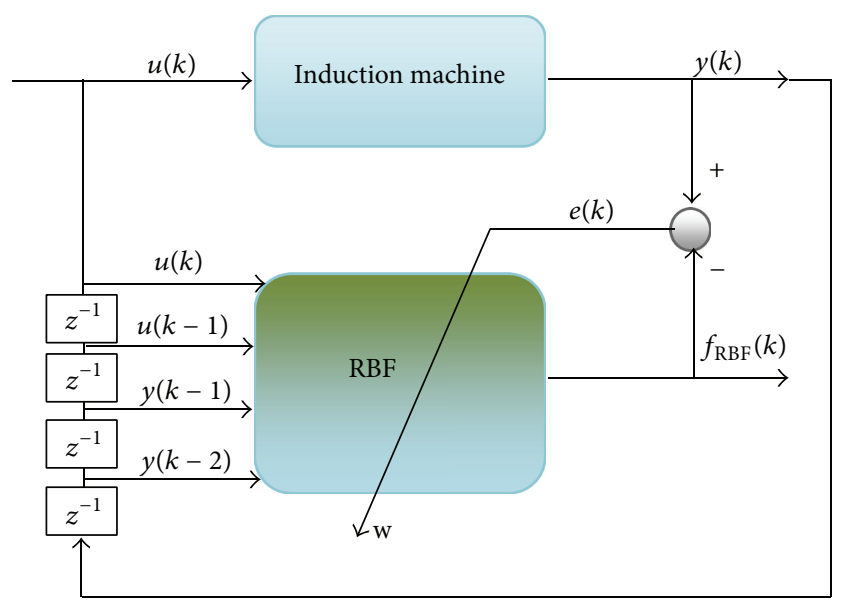

FIGURE 5: Implemented structure for adjusting the weights vector $\mathbf{w}$.

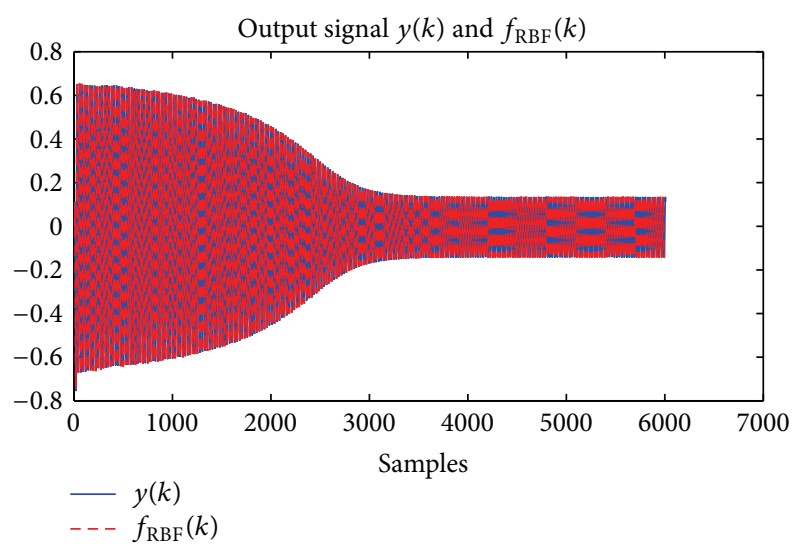

FIGURE 6: Validation model.

3.2. Simulations. Figure 6 is a comparison between the outputs obtained in the plant with the outputs obtained from the RBF network from the structure shown in Figures 6 and 7.

The RBF network training process was based in the adjustment of the basis functions parameters from the input data and the centers group; the weights vector was found using the least squares method. After training the network we obtained the best results with the following parameters:

$$
\begin{array}{ccc}
\text { Radius }(\sigma) & \begin{array}{c}
\text { Mean Square error } \\
J=\mathbf{e}^{\mathrm{T}} \mathbf{e}
\end{array} \\
\sigma=0,75657 \quad e=3,085 e-006 \quad \mathrm{~N}^{\circ} \text { Centers }=100 .
\end{array}
$$

From now on we must be careful not to use the same data used in the training where $40 \%$ of samples were used for validation. Since the identified system is nonlinear, both tests have been executed with the system operating in similar conditions, that is, with the same weights group obtained during the training phase.

Once the system is identified and the model validated, the data obtained from the plant can be ignored; we begin to analyze its dynamic testing the structure shown in Figure 8.

The model main attributes are as follows: 


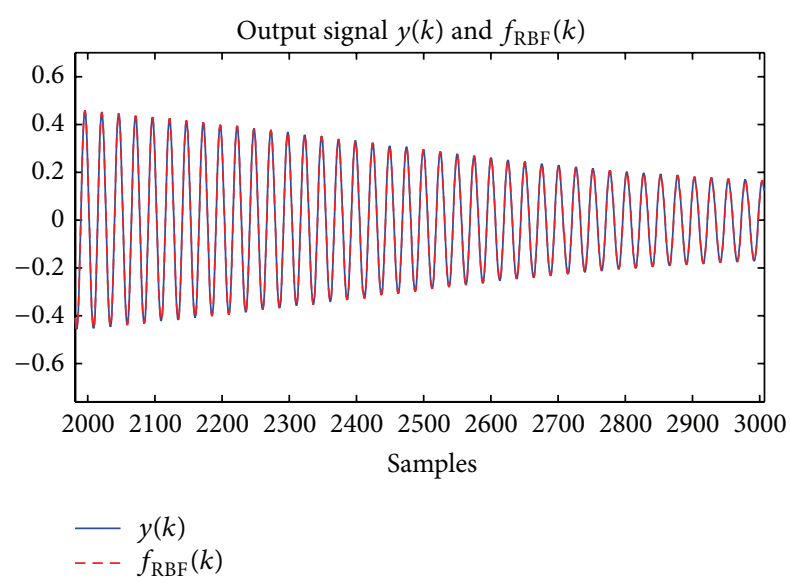

FIGURE 7: The graphic has been amplified in the discrete time region 2000 to 3000 , where blue line represents the plant output and the red line the RBF output.

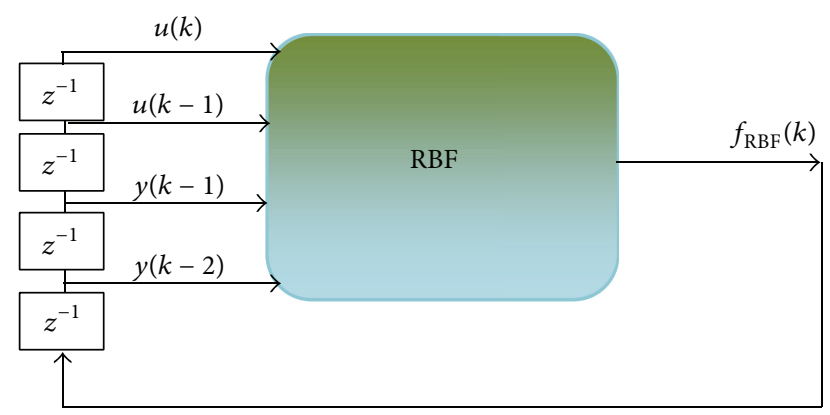

FIGURE 8: Dynamic analysis of the plant from a RBF network.

(i) possibility of representing the nonlinear behavior of the phenomena;

(ii) approximate an input/output mapping;

(iii) capacity of learning, which was obtained through a training session with input/output representative examples;

(iv) capacity of generalization, which allows the RBF to have a satisfactory performance in response to data that do not belong to the training group.

(v) tolerance to errors, which allows the RBF to continue presenting acceptable results in case of data noise.

The model presented in Figure 8 is generally referred to as a model of recursive prediction, in other words, neurodynamics model with associative memories (present and past inputs) and input/output mapping. We can describe the model as

$$
\begin{gathered}
\mathbf{x}(k+1) \cong f_{\mathrm{RBF}}(\mathbf{x}, \mathbf{c}, \mathbf{w}, \sigma) \\
=f[u(k), u(k-1), y(k-1), \\
y(k-2), \mathbf{c}, \mathbf{w}, \sigma] .
\end{gathered}
$$

The notions of states perform an essential role in the mathematical formulation of dynamic systems; that is, the notions of states perform an essential role in the mathematical formulation of dynamic systems. A state represents the actual quantity of information about the effect of the past inputs applied to the system, where this information is enough to describe its future evolution. The RBF viewed as a nonlinear dynamic system, with particular emphasis on the stability problem, is referred to as a neurodynamics model [13]. If we consider the model described in (25) as a model of states and we add and subtract a constant and positive parameter $a$. We have

$$
\begin{aligned}
\mathbf{x}(k+1) & =-a \mathbf{x}(k)+\mathbf{a} x(k)+f(\mathbf{x}(k), \mathbf{u}(k)) \\
& =-a \mathbf{x}(k)+\mathbf{h}(\mathbf{x}(k), \mathbf{u}(k)),
\end{aligned}
$$

where $\mathbf{h}(\mathbf{x}(k), \mathbf{u}(k))=\mathbf{a} x(k)+f(\mathbf{x}(k), \mathbf{u}(k))$ so we can say that the RBF network estimated states can be described as

$$
\widehat{\mathbf{x}}(k+1)=-a \widehat{\mathbf{x}}(k)+\widehat{h}\left(\mathbf{x}, \mathbf{u}, \mathbf{c}_{i}, \sigma\right), \quad \widehat{x}(0)=\widehat{x}_{0} .
$$

Since $\widehat{x}_{i}$ are the states of the RBF, $c_{i}$, and $\sigma$ the adjustment parameters and considering the function $\widehat{h}$ as

$$
\widehat{h}(\mathbf{x}, \mathbf{u}, \mathbf{c}, \sigma)=\sum_{j=1}^{M} w_{i} \varphi\left(\mathbf{x}, \mathbf{u}, \mathbf{c}_{i}, \sigma\right),
$$

assuming we know the ideal weights, we have

$$
\widehat{\mathbf{x}}(k+1)=-a \widehat{\mathbf{x}}(k)+\sum_{j=1}^{M} w_{i} \varphi\left(\mathbf{x}, \mathbf{u}, \mathbf{c}_{i}, \sigma\right) ;
$$

if we consider $a=-1$, we have

$$
\widehat{\mathbf{x}}(k+1)=\widehat{\mathbf{x}}(k)+\sum_{j=1}^{M} w_{i} \varphi\left(\mathbf{x}, \mathbf{u}, \mathbf{c}_{i}, \sigma\right) ;
$$

considering the Gaussian radial basis function, we will have the following representation:

$$
\widehat{\mathbf{x}}(k+1)=\widehat{\mathbf{x}}(k)+\mathbf{w}(k) \sum_{i=1}^{M} \exp \left[-\frac{1}{2 \sigma^{2}}\left(\mathbf{x}-\mathbf{c}_{i}\right)^{T}\left(\mathbf{x}-\mathbf{c}_{i}\right)\right] .
$$

In the previous equation the values $\mathbf{x}$ are called estimated states and its equations differences the quantities that each moment characterizes the phases or the system evolution. Now we can extend the mentioned concepts to a more interesting geometrical representation, the vector field.

A vector field in $\mathbb{R}^{n}$ is a function $\mathbf{F}: D \subset \mathbb{R}^{n} \rightarrow \mathbb{R}^{n}$, defined in a subset $D$, where every point $x$ in $D$ is associated with a vector $\mathbf{F}(\mathbf{x})$ in $\mathbb{R}^{n}$. The most interesting geometrical representation of a vector field is the one that represents $\mathbf{F}(\mathbf{x})$ as a vector with initial extremity in $x$ for each point $x$ in $D$ [25]. To construct the system's vector field we can consider the following system:

$$
\begin{aligned}
\Delta x_{1}(k) & =x_{1}(k+1)-x_{1}(k) \\
& =f_{1}\left(x_{1}(k), x_{2}(k), u(k)\right)-x_{1}(k), \\
\Delta x_{2}(k) & =x_{2}(k+1)-x_{2}(k) \\
& =f_{2}\left(x_{1}(k), x_{2}(k), u(k)\right)-x_{2}(k),
\end{aligned}
$$




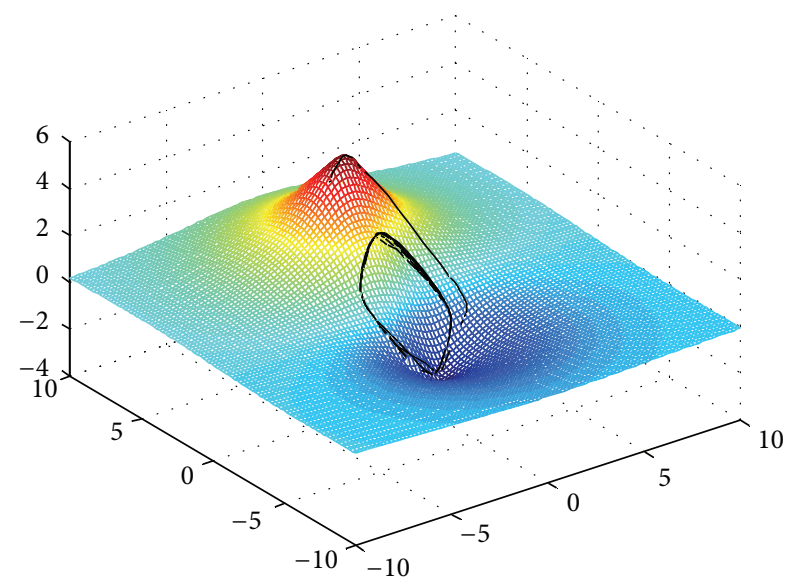

FIGURE 9: The Van der Pol equations diagram (in black) and the RBF basin of attraction.

where $\Delta=x(k+1)-x(k)$ is the directional variation of the point in a plane, and the plane determined by these two variables is called phase portrait. For the RBF, we can define it as

$$
\begin{aligned}
& \mathbf{x}_{1}(k+1)=f_{1}\left(\mathbf{x}_{1}, \mathbf{x}_{2}\right) \\
& \mathbf{x}_{2}(k+1)=f_{\mathrm{RBF}}\left(\mathbf{x}_{1}, \mathbf{x}_{2}, \mathbf{w}, \mathbf{c}, \mathbf{u}, \sigma\right) .
\end{aligned}
$$

The main goal is to present the RBF response to different systems (nonlinear) and analyze the graphics. First, the RBF network was trained and simulated in order to obtain the dynamical solution with basin of attraction and equilibrium point for known system. To analyze its behavior and response the Van der Pol oscillator (Nonlinear System with Limit Cycle) was used:

$$
\begin{gathered}
\mathbf{x}_{1}(k+1)=\mathbf{x}_{2} \\
\mathbf{x}_{2}(k+1)=-\mu\left(\mathbf{x}_{1}^{2}-1\right) \mathbf{x}_{2}-\mathbf{x}_{1} .
\end{gathered}
$$

Identifying the nonlinear system above and considering $\mu=1$, we obtained the following response using a RBF.

Figure 9 shows the system dynamic along the estimated orbit of the RBF approximations in the dynamic system that can be seen as a stable limit cycle of periodic orbit, stable in the sense that nearby trajectories approach it asymptotically. The attractors represent only the equilibrium states of a dynamic system that was observed experimentally. In the Following, we have the phase diagram plot (the real dynamic system, the current plant of the induction motor).

In Figure 11 we can observe the system nonlinearity region. For better visualization a straight line intercepting the phase diagram (Figure 10 rotated) has been used to put in evidence the nonlinearity of the system.

Once the RBF was trained and the model validated for the unknown system (real dynamic system, the current plant of the induction machine), the parameters (weights) were obtained and the structure shown in Figure 8 was used to analyze all the process dynamic. The RBF networks response shown below is presented along with the current plant phase diagram (in blue) in Figure 10.

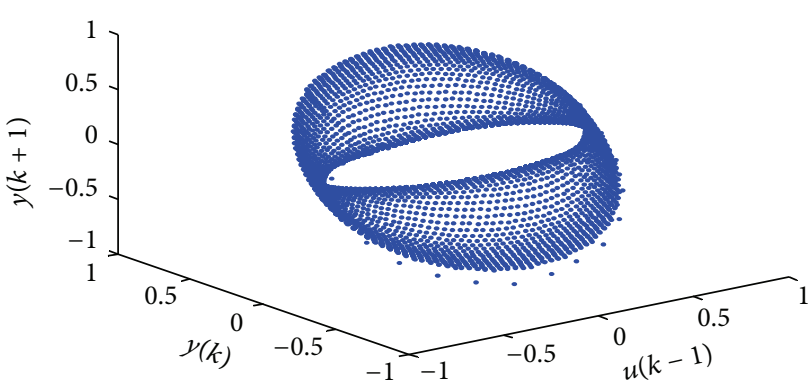

FIGURE 10: Induction machine current plant phase diagram (the orbit).

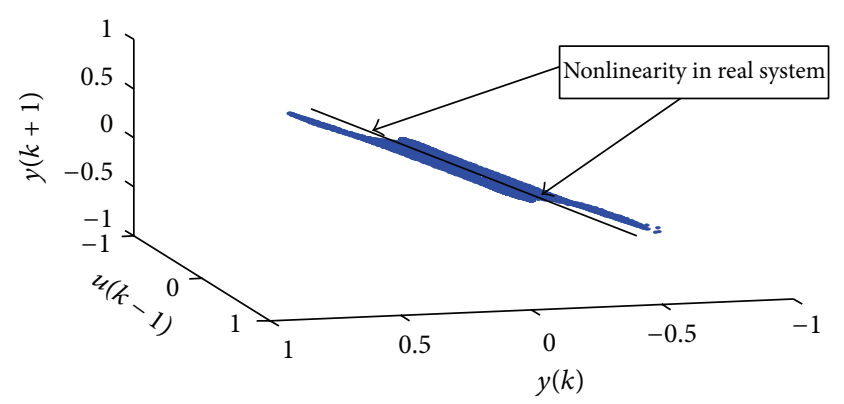

FIgURE 11: The nonlinearity region of the system.

In Figure 14, a vector sequence in space can be represented as a trajectory of the system or the model immersed in the space of states created by the RBF network. In Figures, 12,13 , and 14 , we show that a RBF network take the form of a nonlinear dynamic equation that manipulates the location of the attractors in a structure of interest. It is possible to establish a relationship between the physics of the electrical current of the induction machine and the algorithms of the computation (RBF) [13].

\section{Conclusions}

In this paper, we presented a mathematical tool and implemented it to effectively resolve a problem in modern engineering: the plant modeling, supposedly unknown and with significant nonlinear attributes. The RBF networks dynamic behavior was tested and analyzed from data obtained from the induction motor current plant, without provoking the system instability at the same time optimizing some formulated performance standards using mathematical tools that can be algebraically manipulated.

This paper describes the Gaussian radial basis function analysis and synthesis in a typical problem of functions approximation. A peculiar characteristic of these RBFs is the fact that they are universal approximators with a simple architecture and capable of representing complex nonlinear mappings using nonlinear basis functions and adjustment parameters. The RBF networks can be seen as a mathematical structure of multivariate approximation that are frequently applied in modern approximation theory; what makes them 


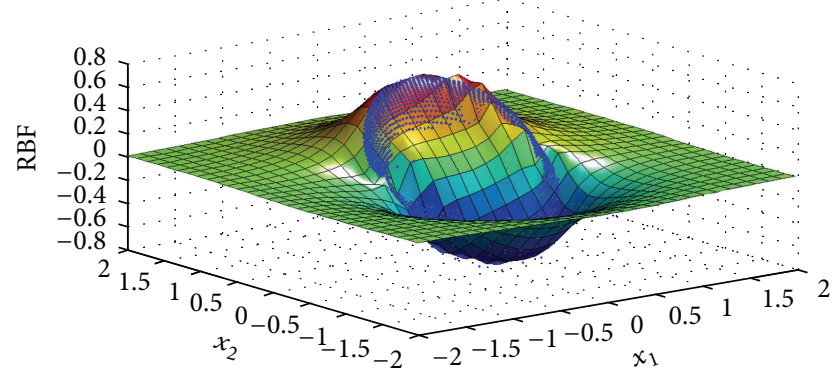

FIGURE 12: The RBF attraction region with the system dynamic.

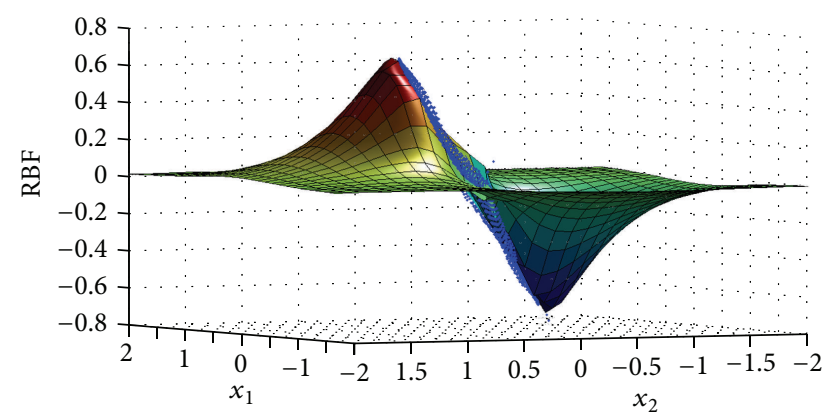

Figure 13: The nonlinearity region of the system dynamics (Figure 12 rotated).

a tool that can be used in the modeling and identification of nonlinear systems.

The contributions of this paper are primarily focused in the result presented and in the RBF network training and simulation for obtaining the dynamical solution with basin of attraction and equilibrium point for known and unknown system. Also a relationship between the physics of the machine, the Van der Pol equations, and the RBF response was established. This technique can be used in other related areas such as dynamic fault diagnosis and dynamical pattern recognition. The observation errors are taken as the measure of similarity between the test and training dynamical patterns.

\section{Conflict of Interests}

The authors declare that there is no conflict of interests regarding the publication of this paper.

\section{References}

[1] M. NØrgaard, O. Ravn, N. K. Poulsen, and L. K. Hansen, Neural Networks for Modeling and Control of Dynamic Systems, Springer, London, UK, 2003.

[2] L. A. Aguirre, Introdução à Identificação de Sistemas: Técnicas Lineares e Não Lineares Aplicadas a Sistemas Reais, Editora da UFMG, Belo Horizonte, Brasil, 3rd edition, 2007.

[3] D. S. Broomhead and D. Lowe, "Multivariable functional interpolation and adaptive networks," Complex Systems, vol. 2, no. 3, pp. 321-355, 1988.

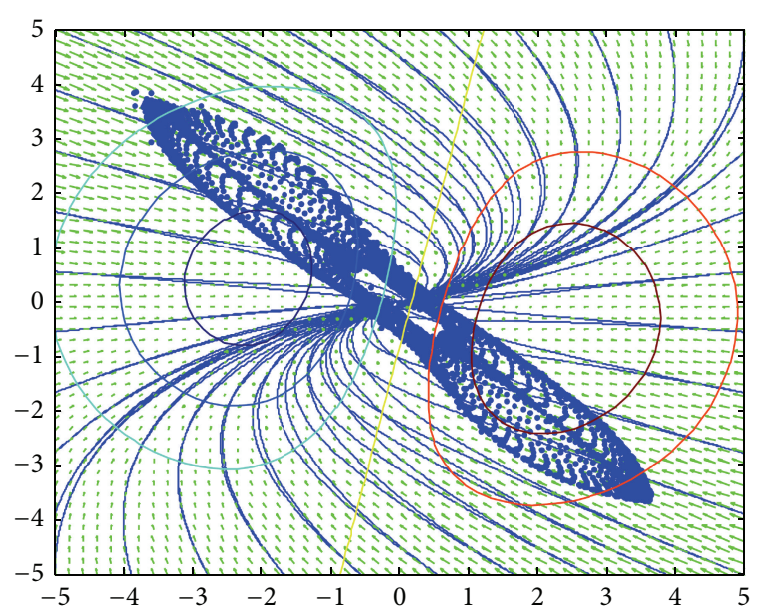

FIGURE 14: The RBF phase portrait with a domain of attraction of a dynamic system identified.

[4] J. Moody and C. J. Darken, "Fast learning in networks of locallytuned processing units," Neural Computation, vol. 1, no. 2, pp. 281-294, 1989.

[5] T. Poggio and F. Girosi, "Networks for approximation and learning," Proceedings of the IEEE, vol. 78, no. 9, pp. 1481-1497, 1990.

[6] T. Poggio and F. Girosi, "Regularization algorithms for learning that are equivalent to multilayer networks," Science, vol. 247, no. 4945, pp. 978-982, 1990.

[7] M. G. A. Nassef, C. Schenck, and B. Kuhfuss, "Simulation-based parameter identification of a reduced model using neural networks," in Proceedings of the 9th IEEE International Conference on Control and Automation (ICCA '11), pp. 974-978, Santiago, Chile, December 2011.

[8] C.-N. Ko, "Identification of non-linear systems using radial basis function neural networks with time-varying learning algorithm," IET Signal Processing, vol. 6, no. 2, pp. 91-98, 2012.

[9] S. Lyu, C. Wu, and S. Zhang, "Application of the RBF method to the estimation of temperature on the external surface in laminar pipe flow," Mathematical Problems in Engineering, vol. 2013, Article ID 205169, 11 pages, 2013.

[10] C. M. Bishop, Pattern Recognition and Machine Learning, Springer, New York, NY, USA, 2006.

[11] M. D. Buhmann, Radial Basis Functions: Theory and Implementations, Cambridge University Press, Cambridge, UK, 2003.

[12] G. E. Fasshauer, Meshfree Approximation Methods with MAT$L A B$, vol. 6 of Interdisciplinary Mathematical Sciences, Mainland Press, Singapore, 2008.

[13] S. Haykin, Neural Networks and Learning Machines ? Prentice Hall, Upper Saddle River, NJ, USA, 2nd edition, 2009.

[14] J. Lin and Y. Liu, "Efficient gradient descent method of RBF neural networks with adaptive learning rate," Journal of Electronics, vol. 19, no. 3, pp. 255-258, 2002.

[15] C. Li and G. Shi, "Weights optimization for multi-instance multi-label RBF neural networks using steepest descent method," Neural Computing and Applications, vol. 22, no. 7-8, pp. 1563-1569, 2013.

[16] V. N. Vapnik, The Nature of Statistical Learning Theory, Springer, New York, NY, USA, 2nd edition, 2000. 
[17] T. Hastie, R. Tibshirani, and J. Friedman, The Elements of Statistical Learning: Data Mining, Inference, and Prediction, Springer, New York, NY, USA, 2001.

[18] B. W. Silverman, Density Estimation for Statistics and Data Analysis, Chapman \& Hall, London, UK, 1986.

[19] W. Yao, X. Chen, Y. Zhao, and M. V. Tooren, "Concurrent subspace width optimization method for RBF neural network modeling," IEEE Transactions on Neural Networks and Learning Systems, vol. 23, no. 2, pp. 247-259, 2012.

[20] S.-K. Oh, W.-D. Kim, W. Pedrycz, and S.-C. Joo, "Design of Kmeans clustering-based polynomial radial basis function neural networks (pRBF NNs) realized with the aid of particle swarm optimization and differential evolution," Neurocomputing, vol. 78, no. 1, pp. 121-132, 2012.

[21] X. Zong-yi, Q. Yong, P. Xue-miao, J. Li-min, and Z. Yuan, "Modelling of the automatic depth control electrohydraulic system using RBF neural network and genetic algorithm," Mathematical Problems in Engineering, vol. 2010, Article ID 124014, 16 pages, 2010.

[22] J. A. Riveros, A. G. Yepes, F. Barrero et al., "Parameter identification of multiphase induction machines with distributed windings-part 2: time-domain techniques," IEEE Transactions on Energy Conversion, vol. 27, no. 4, pp. 1067-1077, 2012.

[23] M. J. D. Powell, "Radial basis functions for multivariable interpolation: a review," in Algorithms for the Approximation of Functions and Data, pp. 143-167, Oxford University Press, Oxford, UK, 1987.

[24] H. Wendland, Scattered Data Approximation, Cambridge University Press, Cambridge, UK, 2005.

[25] G. F. Simmons, Differential Equations: Theory, Technique, and Practice, McGraw-Hill, New York, NY, USA, 2006. 


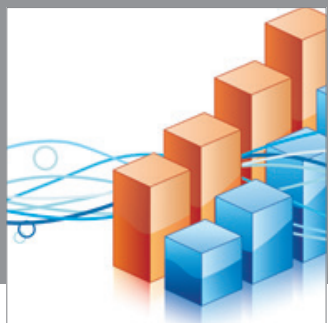

Advances in

Operations Research

mansans

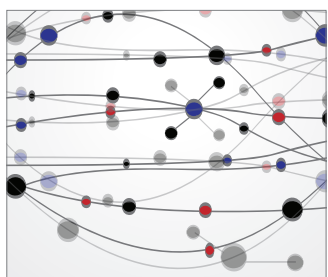

The Scientific World Journal
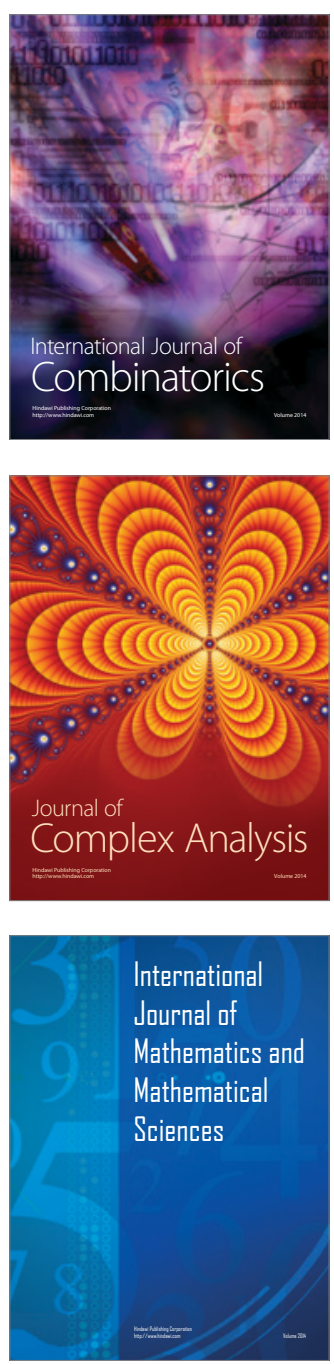
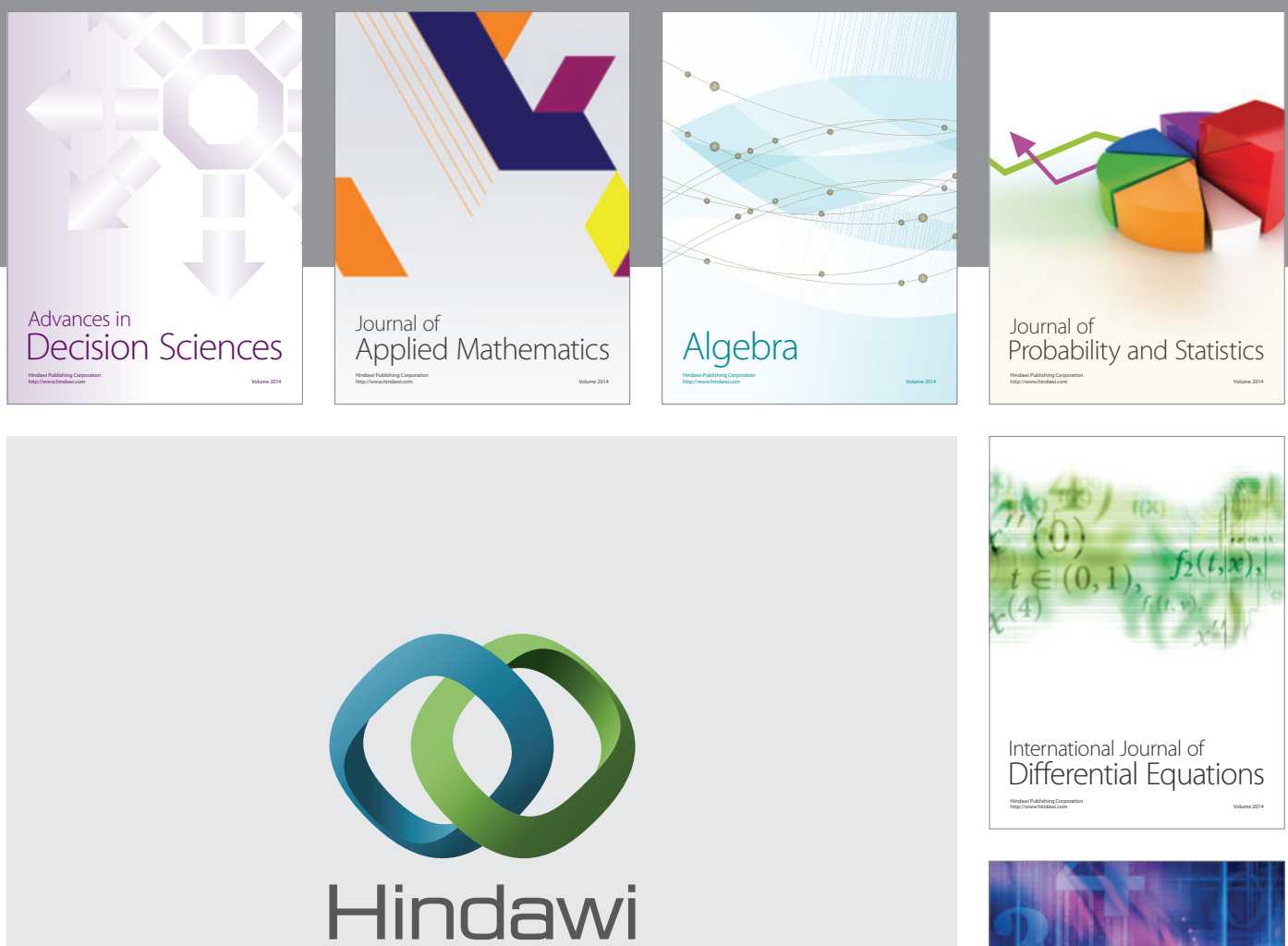

Submit your manuscripts at http://www.hindawi.com
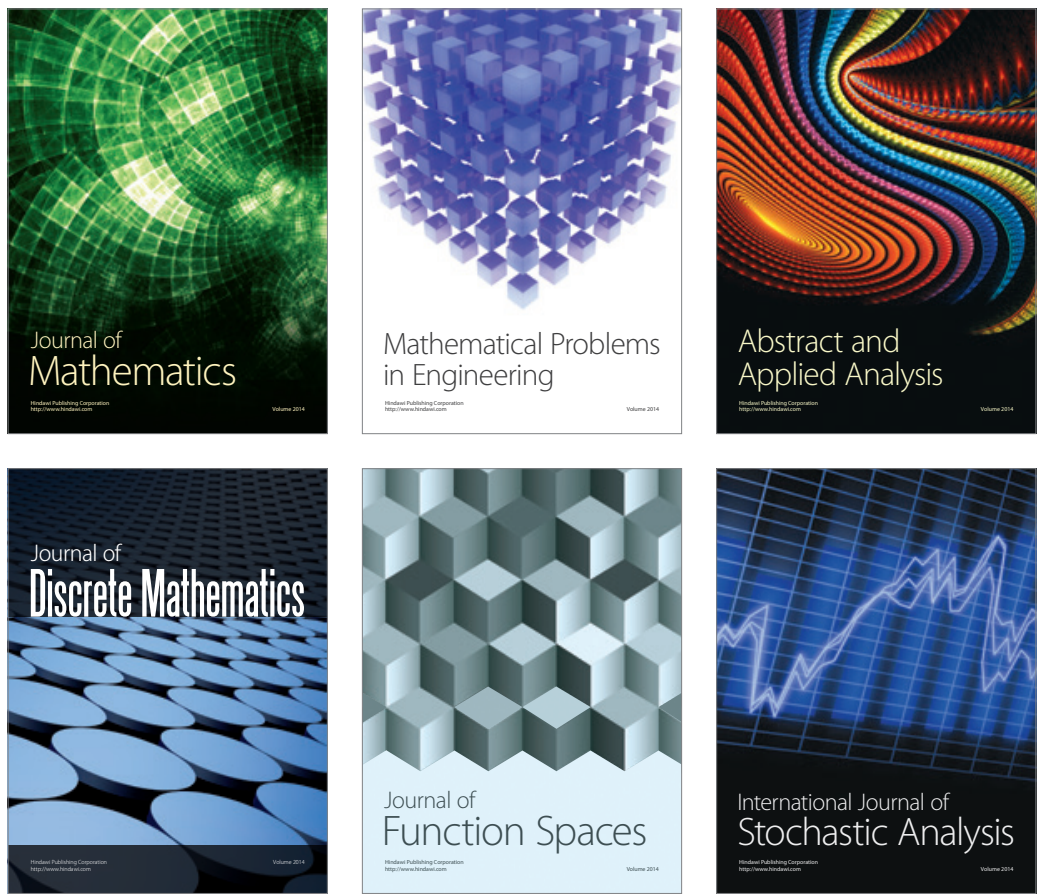

Journal of

Function Spaces

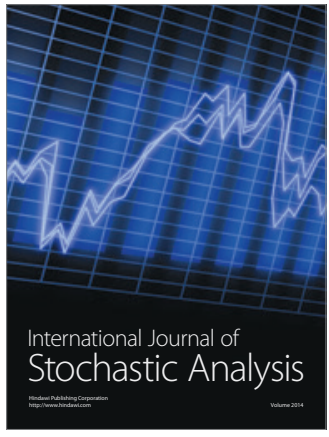

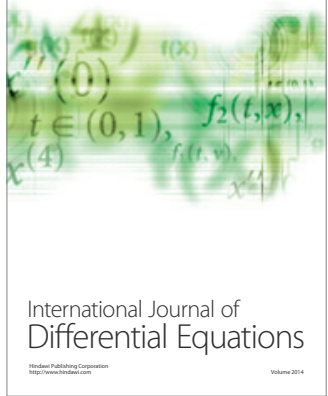
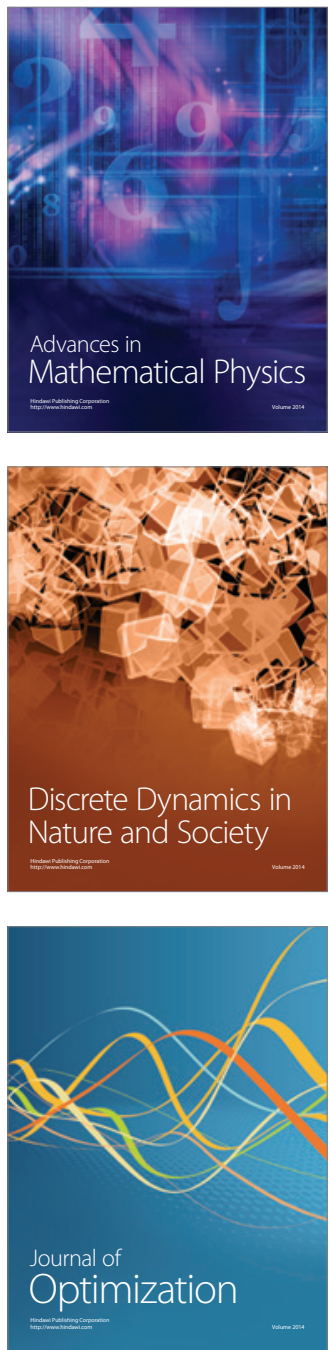